\title{
CRESCIMENTO DAS MUDAS DE CUPUAÇU (Theobroma grandiflorum) SOB EFEITO DE DIFERENTES COMPOSIÇÕ̃S DE BIOFERTILIZANTES
}

\author{
Ediana Pereira NASCIMENTO ${ }^{1}$ \\ Milton César Costa CAMPOS ${ }^{2} *$ \\ Leandro Coutinho $\mathrm{ALHO}^{3}$ \\ Douglas Marcelo Pinheiro SILVA ${ }^{2}$ \\ Felipe Costa WECKHER ${ }^{4}$ \\ Bruno Campos MANTOVANELLI ${ }^{5}$ \\ Jose Mauricio da CUNHA
}

\begin{abstract}
${ }^{1}$ Engenheira Agrônoma da Universidade Federal do Amazonas- UFAM, ediana.agronomia@ gmail.com
$*^{2}$ Engenheiro Agrônomo, Professor Dr. Adjunto IV, Bolsista de Produtividade do CNPq - Universidade Federal do Amazonas - UFAM - Campus de Humaitá, e-mail: mcesarsolos@ gmail.com;

${ }^{3}$ Mestre em Agronomia Tropical. Docente do Instituto Federal de Ciência, Inovação e Tecnologia do Amazonas-IFAM, leandro_alho@yahoo.com

${ }^{4}$ Academico de Engenheira Agrônoma da Universidade Federal do Amazonas- UFAM, felipec.weckner@gmail.com

${ }^{5}$ Engenheiro Agrônomo, Mestrando pelo Programa de Pós Graduação em Ciência do Solo da Universidade Federal Rural de Pernambuco - UFRPE - Recife, e-mail: brunomantovanelli21 @ gmail.com.

${ }^{6}$ Bacharel em Física, Professor Doutorando do Programa de Pós Graduação em Física Ambiental Adjunto I Universidade Federal do Amazonas - UFAM - Campus de Humaitá, e-mail: maujmc@gmail.com.
\end{abstract}

Recebido em: 04/09/2016 - Aprovado em: 12/12/2017 - Disponibilizado em: 30/12/2017

\section{RESUMO:}

O cupuaçuzeiro (Theobroma grandiflorum) é uma das espécies da Amazônia com maior potencial para fruticultura, ocorre naturalmente em regiões de várzeas férteis não inundáveis localizadas no interior de matas primárias. O presente trabalho objetivou avaliar o crescimento de mudas de cupuaçu (Theobroma grandiflorum) sob efeito da aplicação de diferentes composições de biofertilizantes. O experimento foi conduzido no viveiro do Instituto de Educação Agricultura e Ambiente da Universidade Federal do Amazonas, situado no município de Humaitá, AM. Foram preparados 12 composições de biofertilizantes, em galões de 20 L, utilizando-se diferentes concentrações de esterco bovino e/ou caroços de açaí triturados e/ou palha de arroz. Após o preparo dos biofertilizantes, os mesmos foram quantificados quimicamente. O delineamento foi em blocos casualizados com 12 tratamentos e uma testemunha, com 4 repetições, totalizando 208 unidades experimentais. As mudas foram avaliadas quanto ao número de folhas, diâmetro do caule, altura da planta, tamanho da raiz, peso seco da parte aérea, da raiz e total. Os resultados foram submetidos à análise de variância (ANOVA) e, quando significativo, comparados pelo teste de Scott-Knott a $5 \%$ de probabilidade. Todas as variáveis analisadas apresentaram os maiores valores quando submetidos aos tratamentos 01 e 12 . Não houve diferença significativa entre os tratamentos quando relacionados ao peso seco da raiz. Os biofertilizantes com predominância de esterco bovino, seguidos da composição de caroços de açaí triturados e palha de arroz favoreceram o maior crescimento das mudas de cupuaçu.

Palavras chave: Produção de muda, resíduos vegetais, fertirrigação.

\section{GROWTH OF SEEDLINGS OF CUPUAÇU (Theobroma grandiflorum) UNDER THE EFFECT OF DIFFERENT COMPOSITIONS OF BIOFERTILIZERS}

\begin{abstract}
ABSTTRACT:
The cupuaçu (Theobroma grandiflorum) is a species of the Amazon with the greatest potential for fruit growing naturally occurs in areas not flood fertile floodplains located within primary forests. This study aimed to evaluate the growth of seedlings of cupuaçu (Theobroma grandiflorum) under effect of different compositions of biofertilizers. The experiment was conducted in the nursery of the Institute of Agriculture and Environmental Education at the Federal University of Amazonas, in the municipality of Humaitá - AM. Biofertilizers 12 compositions were prepared in 20 gallons of $\mathrm{G}$, using different concentrations of manure and / or lumps acai broken and / or rice straw. After preparation
\end{abstract}


of biofertilizers, they were chemically quantified. The design was a randomized block design with 12 treatments and a control, distributed in 4 blocks with 4 replications, totaling 208 experimental units. The seedlings were evaluated on the number of leaves, stem diameter, plant height, root size, dry weight of shoot, root and total. The results were subjected to analysis of variance (ANOVA) and when significant, compared by Scott-Knott test at 5\% probability. All parameters have shown good results when subjected to treatments 01 and 12. Was not significantly different between treatments when related to root dry weight. Biofertilizers with predominance of cattle manure, followed by predominance of lumps of crushed acai and rice straw attributed the better results for seedlings of cupuaçu.

Keywords: Plant propagation, vegetable waste, fertigation.

\section{INTRODUÇÃO}

O cupuaçuzeiro é uma das espécies da Amazônia com maior potencial para fruticultura (Alfaia; Ayres, 2004). Ocorre naturalmente em regiões de várzeas férteis não inundáveis localizadas no interior de matas primárias. É uma espécie arbórea da família Sterculiaceae que, em cultivos comerciais pode variar de 4 a $8 \mathrm{~m}$ de altura. Quando encontrada espontaneamente nas áreas de mata do sul e nordeste da Amazônia Oriental e nordeste do Maranhão, pode atingir até $18 \mathrm{~m}$ de altura (Lorenzi, 2000). Nas últimas décadas, com o aumento da demanda, o cupuaçuzeiro passou por um processo de transição do extrativismo para a forma cultivada, emergindo nos últimos dez com o aumento da área plantada e com a ampliação do cultivo para outras regiões brasileiras (EMBRAPA, 1999).

No plantio comercial de cupuaçu são utilizadas mudas propagadas por sementes ou por via vegetativa, por meio da enxertia. Fruteira perene, a propagação vegetativa do cupuaçuzeiro é uma das opções mais promissoras para a formação de mudas de comprovado valor genético, colaborando para melhoria de sua produtividade (Cruz, 2007; Ferreira et al., 2007). A caracterização das condições mais apropriadas para a produção de mudas desta frutífera propicia melhores condições para o crescimento inicial em campo, colaborando para o aumento da homogeneidade, sanidade e redução da mortalidade do plantio. A produção de mudas é influenciada por fatores internos de qualidade das sementes e fatores externos, como água, luz, temperatura, oxigênio e agentes patogênicos, associados ao tipo de substrato (Brasil, 1992; Nomura et al., 2008).

Para melhorar as características dos substratos têm-se recomendado o uso de substâncias que agreguem qualidade a eles, como os bioestimulantes. Trata-se de produtos alternativos, cuja utilização na agricultura vem crescendo e os custos de produção têm se mostrado economicamente viáveis, já que os mesmos podem ser preparados pelo próprio produtor reduzindo o uso de insumos importados (Deleito et al., 2000). A importância do biofertilizante se deve ao fato de este apresentar uma composição mineral diversa, sendo assimilado mais rapidamente por estar na forma líquida (Barros \& Liberalino Filho, 2008) atuando nutricionalmente sobre o metabolismo das plantas e também na ciclagem de nutrientes e redução da acidez do solo (Galbiatti et al., 1996; Medeiros et al., 2003). De acordo com Cavalcante et al. (2010), o biofertilizante 
contribui para a melhoria da estrutura física do solo, diminui as perdas elevadas de água do substrato por evaporação e aumenta o período de turgescência das células.

Atualmente vários materiais orgânicos e inorgânicos estão sendo utilizados na formulação de biofertilizantes para a produção de mudas, porém há necessidade de se determinar os produtos mais apropriados a fim de atender sua demanda quanto ao fornecimento de nutrientes, propriedades físicas, químicas, físico-químicas e posterior resistência da planta a doenças (Lima et al., 2006). Neste contexto a utilização do esterco bovino, caroços de açaí triturado e palha de arroz podem ser alternativas de substratos. $\mathrm{O}$ esterco bovino normalmente possui grande quantidade de matéria orgânica, o que contribui com as características física, química e biológica do solo, o caroço de açaí triturado é um subproduto do beneficiamento do fruto do açaizeiro, muito comum em grande parte da Amazônia que também pode ser utilizado como adubo. Outro componente que pode ser utilizado na formulação do biofertilizante é a casca de arroz que tem sido muito utilizada pelos agricultores (Carrijo et al., 2002).

Diante do exposto, objetivou-se com o presente trabalho avaliar o crescimento das mudas de cupuaçu (Theobroma grandiflorum) sob efeito de diferentes composições de biofertilizantes.

\section{MATERIAL E MÉTODOS}

O experimento foi conduzido no período de janeiro a junho de 2013 , no viveiro de produção de mudas do Instituto de Educação, Agricultura e Ambiente da Universidade Federal do Amazonas, situado no município de Humaitá, AM, estando situado sob as seguintes coordenadas geográficas de $7^{\circ} 30^{\prime} 56^{\prime}$ ' latitude Sul e $63^{\circ} 1^{\text {' }}$ 35" longitude Oeste. O clima da região, segundo a classificação de Köppen é do tipo tropical chuvoso, temperaturas variando entre 25 e $27{ }^{\circ} \mathrm{C}$ e precipitação média anual de $2.500 \mathrm{~mm}$, com período chuvoso iniciando em outubro e prolongando-se até junho, apresentando umidade relativa do ar entre 85 e $90 \%$.

Para o preparo dos biofertilizantes foram utilizados caroços de açaí e palha de arroz coletados das agroindústrias decompostos e o esterco bovino fresco coletado nas propriedades rurais. Os biofertilizantes foram preparados conforme metodologia de Santos (1992), em galões com capacidade de $20 \mathrm{~L}$, cada biofertilizante foi preparado utilizando-se de diferentes porções dos compostos orgânicos (esterco bovino, caroços de açaí e palha de arroz) perfazendo 4 L de compostos orgânicos por biofertilizante.

O preparo dos biofertilizantes ocorreu em duas etapas, cuja primeira etapa consistiu da adição de $2 \mathrm{~L}$ dos compostos orgânicos em $4 \mathrm{~L}$ de águas, os quais foram homogeneizados e hermeticamente fechados. Após uma semana, foram adicionados os $2 \mathrm{~L}$ restantes 
dos compostos orgânicos, completando-se o volume com água para $20 \mathrm{~L}$ e mantendo-os hermeticamente fechados por mais 60 dias, completando-se, então, a segunda etapa do preparo. Os biofertilizantes produzidos apresentaram as seguintes proporções:

Testemunha: somente água;

Biofertilizante 1: $100 \%$ de esterco bovino; Biofertilizante 2: $75 \%$ de esterco bovino +25 $\%$ de caroços de açaí triturados; Biofertilizante 3: $50 \%$ de esterco bovino +50 $\%$ de caroços de açaí triturados; Biofertilizante 4: $25 \%$ de esterco bovino +75 $\%$ de caroços de açaí triturados; Biofertilizante 5: $0 \%$ de esterco bovino +100 $\%$ de caroços de açaí triturados; Biofertilizante 6: $75 \%$ de esterco bovino +25 $\%$ de palha de arroz; Biofertilizante 7: $50 \%$ de esterco bovino $+50 \%$ de palha de arroz; Biofertilizante 8: $25 \%$ de esterco bovino +75 $\%$ de palha de arroz; Biofertilizante 9: $0 \%$ de esterco bovino $+100 \%$ de palha de arroz; Biofertilizante 10: $0 \%$ de esterco bovino +50 $\%$ de palha de arroz $+50 \%$ de caroços de açaí triturados; Biofertilizante 11: $25 \%$ de esterco bovino $+25 \%$ de palha de arroz $+50 \%$ de caroços de açaí triturados; Biofertilizante 12: $50 \%$ de esterco bovino $+25 \%$ de palha de arroz $+25 \%$ de caroços de açaí triturados.

Após a fabricação dos biofertilizantes foram coletadas amostras para realizar as análises químicas. Os teores de fósforo disponível (P) foram determinados por colorimetria, o potássio $\left(\mathrm{K}^{+}\right)$por fotometria de chama, e o sódio $\left(\mathrm{Na}^{+}\right)$por espectroscopia conforme metodologia de Mackereth, (1978). $\mathrm{O}$ pH em água e em $\mathrm{KCl}$ foi determinado potenciometricamente utilizando-se a metodologia da (EMBRAPA, 2011).

Para determinação do teor de Cálcio $\left(\mathrm{Ca}^{2+}\right)$ e Magnésio $\left(\mathrm{Mg}^{2+}\right)$ foi transferido para um erlenmeyer de $250 \mathrm{~mL}, 100 \mathrm{~mL}$ da amostra, adicionou-se $2 \mathrm{~mL}$ de solução de hidróxido de sódio $(\mathrm{NaOH})$, homogeneizou e adicionou uma pequena quantidade do indicador HHSNN e titulou com solução de EDTA-Na 0,04 M até coloração azul, e anotou o consumo.

$\mathrm{Na}$ obtenção do carbono orgânico foi transferido para um erlenmeyer de $250 \mathrm{~mL}, 50$ $\mathrm{mL}$ da amostra, acrescentou-se $50 \mathrm{~mL}$ de água destilada, $5 \mathrm{~mL}$ de $\mathrm{H}_{2} \mathrm{SO}_{4} 24 \%, 10 \mathrm{~mL}$ de $\mathrm{KMnO}_{4}$ 0,01 $\mathrm{N}$ e levou à ebulição por 10 minutos, retirou da chapa aquecedora e, em seguida, adicionou $10 \mathrm{~mL}$ de ácido oxálico $0,01 \mathrm{~N}$ e titulou com $\mathrm{KMn}_{4}$ 0,01 $\mathrm{N}$ até obtenção da cor rosa forte e anotou-se o volume consumido. Para determinação do Alumínio foi adicionando $1 \mathrm{~mL}$ de $\mathrm{H}_{2} \mathrm{SO}_{4}, 0,02 \mathrm{~N}+1 \mathrm{~mL}$ de ácido ascórbico (homogeneizou) $+10 \mathrm{~mL}$ da solução tampão (homogeneizou e completou para $50 \mathrm{~mL}$ com água destilada, homogeneizou novamente e deixou em repouso por 10 minutos. A cor começou a desaparecer após 15 minutos e as leituras foram efetuadas, esses processos foram realizados por absorção atômica (Malavolta et al., 1989/1997; Santos, 1991).

Como substrato para a formação das mudas foi utilizado um Cambissolo Háplico 
coletado na camada de 0 a $20 \mathrm{~cm}$, o qual foi corrigido com calcário para a elevação do $\mathrm{pH}$ para próximo de 6,0 mantendo-se úmido por 60 dias. As sementes foram coletadas de frutos de cupuaçu sadios e semeadas em sacos de polietileno com capacidade para 3 litros de substrato. Foram semeadas 3 sementes por sacos a uma profundidade de $1,5 \mathrm{~cm}$. Após a emergência foi realizado o desbaste das plantas aos $4 \mathrm{~cm}$ de altura, deixando uma muda por saco. Os tratamentos constituíramse das dozes composições de biofertilizantes e uma testemunha, somando 13 tratamentos. As composições de biofertilizantes testados na produção de mudas de cupuaçu (Theobroma grandiflorum) foram distribuídas em delineamento de blocos casualizados (DBC), distribuídos em 4 blocos, totalizando 208 unidades experimentais.

As aplicações dos biofertilizantes iniciaram após a emergência das plântulas e foram realizados a cada sete dias, utilizando um litro de biofertilizantes diluídos em cinco litros de água, aplicados aos finais de tarde e as avaliações foram feitas quando as mudas estavam com idade de 72 dias e as variáveis avaliadas foram:

Altura da planta (AP) - altura da muda, medida desde o colo da muda até o ápice da última folha com auxílio de uma régua melimetrada;

Número de folhas (NF) - realização da contagem do número de folhas;

Diâmetro do caule (DC) - medido $1 \mathrm{~cm}$ acima do nó formado logo acima da superfície do solo do recipiente, com auxílio de um paquímetro;

Tamanho da raiz (TR) - medidas com o auxílio de uma régua melimetrada;

Peso seco da parte aérea (PSPA), da raiz (PSR) e total (PST) - as mudas foram acondicionadas em saco de papel e colocadas em estufa com circulação forçada de ar à temperatura de $65^{\circ} \mathrm{C}$, até atingirem peso constante, ocasião em que foi procedida à pesagem em balança eletrônica semianalítica. O peso da matéria seca total da planta foi obtido pela soma dos pesos da matéria seca da raiz e da parte aérea.

Os dados dos parâmetros estudados foram submetidos à análise de variância (ANOVA) e quando se alcançou significância estatística mínima de $5 \%$ as médias foram comparadas pelo teste de Scott-Knott a 5\% de probabilidade, utilizando o programa estatístico Statistica (Statistica, 2005).

\section{RESULTADOS E DISCUSSÃO}

De acordo com os dados apresentados na Tabela 1 podemos observar que os maiores valores de $\mathrm{Ca}^{2+}$ e $\mathrm{P}$ disponível ocorreram nos biofertilizantes 01 e 12, com predominância de esterco bovino fresco. Esses resultados assemelham-se com os obtidos por Souza (2003) trabalhando com biofertilizantes enriquecidos com esterco bovino. $\mathrm{O}$ teor de $\mathrm{P}$ disponível dos biofertilizantes 01 e 12 foram de 10,21 e $11,26 \mathrm{mg} \mathrm{L}^{-1}$ respectivamente acima do limite de amplitude considerado ideal para as plantas que segundo Kiehl 
(2002) encontra-se entre 4,4 e 10,2 $\mathrm{mg} \mathrm{L}^{-1}$. Contudo segundo o autor, mesmo os valores estando acima do limite de amplitude estes não afetam o desenvolvimento da cultura uma vez que quando aplicado grande parte fica indisponível para as plantas. Em todos os biofertilizantes o potássio encontrou-se dentro do limite de amplitude de 5,7 $\mathrm{mg} \mathrm{L}^{-1}$ a 42,0 $\mathrm{mg} \mathrm{L}^{-1}$ ideal para o desenvolvimento das plantas como preconizou Kiehl (2002).

Estudando teores de nutrientes em biofertilizantes Darolt (2004) obteve valores similares para os teores de $\mathrm{K}^{+}$e $\mathrm{P}$ disponíveis e pH em água aos desse estudo. No entanto, os valores de $\mathrm{Ca}^{2+}, \mathrm{Mg}^{2+}$, e $\mathrm{Al}^{3+}$ não corroboram com os elevados valores encontrados neste estudo. Essa diferença de valores pode estar relacionada a diferentes composições de matéria prima utilizada no preparo dos biofertilizantes. Vilela (2003) e Araújo (2007) testando biofertilizantes à base de esterco bovino e com complementação de outros compostos e nutrientes encontrou valores elevados para $\mathrm{Ca}^{2+}, \mathrm{Mg}^{2+}, \mathrm{K}^{+}, \mathrm{P}$ disponível, $\mathrm{Na}^{2+}$ e $\mathrm{pH}$ em água assemelhandose com os resultados deste estudo, porém, quando o biofertilizante não foi enriquecido com nutrientes os valores foram baixos.

Tabela 1: Composição química dos biofertilizantes produzidos a partir de esterco bovino, caroço de açaí triturado e palha de arroz na região de Humaitá-AM.

\begin{tabular}{|c|c|c|c|c|c|c|c|c|c|}
\hline Biofertilizantes & $\begin{array}{c}\mathbf{p H} \\
\mathrm{H}_{2} \mathrm{O}\end{array}$ & $\begin{array}{c}\mathbf{p H} \\
\mathrm{KCl}\end{array}$ & $\mathrm{Ca}^{2+}$ & $\mathbf{M g}^{2+}$ & $\mathrm{Al}^{3+}$ & $\mathrm{Na}^{+}$ & $\mathbf{K}^{+}$ & $\mathbf{P}$ & $\mathrm{CO}$ \\
\hline --------------- & - & - & ------------- & ----------- & --------- & ig.L ---.- & 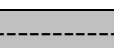 & -------- & $\%$ \\
\hline 01 & 7,37 & 6,63 & 138,05 & 110,00 & 0,38 & 37,60 & 11,98 & 10,21 & 0,42 \\
\hline 02 & 6,23 & 5,21 & 127,25 & 95,35 & 0,24 & 21,02 & 10,65 & 8,50 & 0,49 \\
\hline 03 & 5,17 & 4,98 & 94,05 & 75,39 & 0,13 & 34,60 & 11,06 & 8,52 & 0,80 \\
\hline 04 & 5,87 & 4,90 & 64,90 & 19,05 & 0,17 & 5,86 & 10,04 & 4,98 & 0,83 \\
\hline 05 & 5,06 & 4,29 & 74,06 & 29,05 & 0,18 & 7,89 & 14,04 & 4,08 & 0,71 \\
\hline 06 & 6,78 & 5,08 & 128,05 & 101,8 & 0,38 & 37,50 & 10,98 & 8,21 & 0,25 \\
\hline 07 & 6,93 & 5,36 & 31,47 & 68,09 & 0,31 & 27,91 & 7,83 & 8,27 & 0,24 \\
\hline 08 & 6,81 & 5,11 & 31,08 & 80,24 & 0,12 & 23,17 & 14,64 & 6,08 & 0,25 \\
\hline 09 & 6,35 & 5,45 & 29,01 & 81,02 & 0,10 & 21,31 & 14,5 & 5,14 & 0,38 \\
\hline 10 & 6,49 & 5,19 & 13,80 & 42,35 & 0,09 & 11,04 & 8,36 & 4,18 & 0,38 \\
\hline 11 & 7,00 & 6,19 & 84,96 & 39,05 & 0,08 & 6,86 & 15,04 & 3,08 & 0,20 \\
\hline 12 & 7,02 & 6,09 & 140,30 & 12,15 & 0,14 & 27,09 & 12,28 & 11,26 & 0,21 \\
\hline
\end{tabular}

$\mathrm{Ca}^{2+}:$ Cálcio; $\mathrm{Mg}^{2+}$ : Magnésio; $\mathrm{Al}^{3+}$ : Alumínio trocável; $\mathrm{Na}^{+}$: Sódio; $\mathrm{K}^{+}$: potássio disponível; P: Fósforo disponível; CO: Carbono orgânico.

Os biofertilizantes apresentaram valores de pH em água entre 5,0 e 7,4 considerado como faixa ótima (Tabela 1), concordando com Magrini (2009) que verificou alto valor de $\mathrm{pH}$ em biofertilizantes. Segundo Souza et al. (2005) o valor do pH em água de biofertilizante em geral situa-se entre 5,0 e 8,0 valores inferiores a estes indicam digestão incompleta e superiores apontam retenção excessivamente longa do biofertilizante. $\mathrm{O}$ pH em água foi maior que o $\mathrm{pH}$ em $\mathrm{KCl}$ em todos os biofertilizantes indicando predominância de cargas negativas e nesses casos retendo mais cátions que ânions como por exemplo o cálcio e o magnésio. Brito et al. (2005) relata que o biofertilizante deve possuir um $\mathrm{pH}$ mais 
elevado, pois $\mathrm{pH}$ for muito baixo e o solo estiver um pH ácido acarretará na diminuição do desenvolvimento da planta.

De acordo com as médias obtidas na Tabela 2, todas as variáveis estudadas apresentaram os maiores resultados quando submetidos aos tratamentos 01 e 12 , com a predominância de esterco bovino (Tabela 2), o que pode estar associado aos elevados teores de $\mathrm{Ca}^{2+}, \mathrm{Mg}^{2+}$ trocáveis e $\mathrm{P}$ disponível encontrados nos biofertilizantes.

Tabela 2- Médias referentes a análise de variância das variáveis analisadas de crescimento e desenvolvimento das mudas de cupuaçu (Theobroma grandiflorum) sob efeito de diferentes composições de biofertilizante.

\begin{tabular}{cccccccc}
\hline Tratamentos & $\mathbf{A P}$ & $\mathbf{D C}$ & $\mathbf{N F}$ & $\mathbf{T R}$ & $\mathbf{P S P A}$ & PSR & ST \\
\hline $\mathbf{0 0}$ & $7,95 \mathrm{c}$ & $1,20 \mathrm{~d}$ & $1,93 \mathrm{~d}$ & $2,58 \mathrm{c}$ & $5,53 \mathrm{~b}$ & $6,63 \mathrm{a}$ & $9,43 \mathrm{~b}$ \\
$\mathbf{0 1}$ & $20,95 \mathrm{a}$ & $3,62 \mathrm{a}$ & $7,62 \mathrm{a}$ & $19,20 \mathrm{a}$ & $15,95 \mathrm{a}$ & $8,59 \mathrm{a}$ & $16,12 \mathrm{a}$ \\
$\mathbf{0 2}$ & $15,86 \mathrm{~b}$ & $2,51 \mathrm{~b}$ & $3,68 \mathrm{c}$ & $2,96 \mathrm{c}$ & $6,57 \mathrm{~b}$ & $8,03 \mathrm{a}$ & $16,01 \mathrm{a}$ \\
$\mathbf{0 3}$ & $19,48 \mathrm{a}$ & $2,53 \mathrm{~b}$ & $4,93 \mathrm{~b}$ & $10,56 \mathrm{~b}$ & $8,58 \mathrm{~b}$ & $8,28 \mathrm{a}$ & $10,80 \mathrm{a}$ \\
$\mathbf{0 4}$ & $20,06 \mathrm{a}$ & $2,87 \mathrm{~b}$ & $5,18 \mathrm{~b}$ & $17,10 \mathrm{a}$ & $10,85 \mathrm{~b}$ & $9,21 \mathrm{a}$ & $10,15 \mathrm{a}$ \\
$\mathbf{0 5}$ & $20,81 \mathrm{a}$ & $2,89 \mathrm{~b}$ & $5,12 \mathrm{~b}$ & $10,55 \mathrm{~b}$ & $9,60 \mathrm{~b}$ & $8,75 \mathrm{a}$ & $8,72 \mathrm{~b}$ \\
$\mathbf{0 6}$ & $20,23 \mathrm{a}$ & $3,04 \mathrm{~b}$ & $5,56 \mathrm{~b}$ & $7,50 \mathrm{~b}$ & $6,43 \mathrm{~b}$ & $10,45 \mathrm{a}$ & $8,11 \mathrm{~b}$ \\
$\mathbf{0 7}$ & $19,89 \mathrm{a}$ & $2,62 \mathrm{~b}$ & $5,18 \mathrm{~b}$ & $9,85 \mathrm{~b}$ & $8,54 \mathrm{~b}$ & $8,41 \mathrm{a}$ & $9,15 \mathrm{~b}$ \\
$\mathbf{0 8}$ & $13,79 \mathrm{~b}$ & $2,20 \mathrm{c}$ & $4,18 \mathrm{c}$ & $4,62 \mathrm{c}$ & $6,43 \mathrm{~b}$ & $10,31 \mathrm{a}$ & $8,23 \mathrm{~b}$ \\
$\mathbf{0 9}$ & $12,80 \mathrm{~b}$ & $1,90 \mathrm{c}$ & $3,25 \mathrm{c}$ & $5,70 \mathrm{c}$ & $7,61 \mathrm{~b}$ & $8,00 \mathrm{a}$ & $9,78 \mathrm{~b}$ \\
$\mathbf{1 0}$ & $11,66 \mathrm{~b}$ & $1,74 \mathrm{c}$ & $2,75 \mathrm{~d}$ & $6,02 \mathrm{c}$ & $4,96 \mathrm{~b}$ & $8,31 \mathrm{a}$ & $9,05 \mathrm{~b}$ \\
$\mathbf{1 1}$ & $11,39 \mathrm{~b}$ & $2,37 \mathrm{~b}$ & $4,06 \mathrm{c}$ & $8,62 \mathrm{~b}$ & $7,86 \mathrm{~b}$ & $8,56 \mathrm{a}$ & $0,78 \mathrm{a}$ \\
$\mathbf{1 2}$ & $23,19 \mathrm{a}$ & $3,74 \mathrm{a}$ & $8,00 \mathrm{a}$ & $19,37 \mathrm{a}$ & $17,27 \mathrm{a}$ & $8,50 \mathrm{a}$ & $16,79 \mathrm{a}$ \\
\hline $\mathbf{C V}(\mathbf{\%})$ & $\mathbf{6 , 8 2}$ & $\mathbf{8 , 4 7}$ & $\mathbf{9 , 0 1}$ & $\mathbf{6 , 0 1}$ & $\mathbf{8 , 0 1}$ & $\mathbf{9 , 5 3}$ & $\mathbf{8 , 7 9}$ \\
\hline
\end{tabular}

AP: altura das plantas, DC: diâmetro do caule, NF: número de folhas, TR: tamanho da raiz, PSPA: peso seco parte aérea, PSR: peso seco da raiz e PST: peso seco total.

Média seguida de mesma letra na coluna não difere estatisticamente entre si pelo teste Scott-Knott a $5 \%$ de probabilidade.

Com relação às variáveis tamanho da raiz e peso seco parte aérea os tratamentos que obtiveram os melhores desempenhos foram respectivamente o 01 e 12 constituídos predominantemente por esterco bovino fresco o que de acordo com Barbosa (2004) pode estar relacionado com o alto teor de $\mathrm{Ca}^{2+}$ nos biofertilizantes destas composições o qual influencia diretamente na formação do sistema radicular das plantas e o tratamento 04 cuja formulação é de $25 \%$ de esterco bovino e $75 \%$ de caroços de açaí triturados o que indica que este último composto tem efeito semelhante no crescimento da raiz e no peso seco da parte aérea comparado ao esterco bovino fresco. A variável peso seco da raiz não diferiu entre si em relação aos diferentes tratamentos Tabela 2.

A Tabela 3 apresenta um resumo das análises de variância do experimento, no geral os coeficientes de variação (CV \%) apresentaram valores baixos afirmando a precisão do experimento. Apesar da adubação via foliar não substituir totalmente o fornecimento de adubos no solo para a maioria das culturas, observou-se que as mudas de cupuaçu apresentaram potencial para serem fertilizadas por via foliar com aplicações de biofertilizante. Corroborando com Camargo e Silva (2005) que concluíram haver possibilidade de algumas culturas serem capazes de ser mantidas fertilizadas exclusivamente por via foliar. 
Tabela 3- Análises de variância pelo quadrado médio referentes aos valores de AP: altura das plantas; DC: diâmetro do caule; NF: número de folhas; TR: tamanho da raiz; PSPA: peso seco parte aérea; PSR: peso seco da raiz e PST: peso seco total em função das diferentes doses de biofertilizantes.

\begin{tabular}{ccccccccc}
\hline & \multicolumn{10}{c}{ QUADRADOS MÉDIOS } \\
\hline FV & GL & AP & DC & NF & TR & PSPA & PSR & PST \\
Tratamento & 12 & $364,41^{*}$ & $6,89^{*}$ & $37,87^{*}$ & $529,64^{*}$ & $188,22^{*}$ & $53,44^{\text {ns }}$ & $91,48^{*}$ \\
Bloco & 3 & $364,78^{\text {ns }}$ & $0,48^{\text {ns }}$ & $4,63^{\text {ns }}$ & $44,09^{\text {ns }}$ & $48,34^{\text {ns }}$ & $46,8582^{\text {ns }}$ & $20,89^{\text {ns }}$ \\
Resíduo & 192 & 38,17 & 0,93 & 5,05 & 28,86 & 27,00 & 56,5787 & 9,22 \\
\hline CV (\%) & & 6,82 & 8,47 & 9,01 & 6,01 & 8,01 & 9,53 & 8,79
\end{tabular}

$\mathrm{FV}=$ fonte de variação; GL = graus de liberdade; $\mathrm{CV}=$ Coeficiente de variação \%; ns = não significativo ao teste de Skot-Knot; *= significativos para o nível de $5 \%$ de probabilidade para o teste F.

\section{CONCLUSÕES}

Os tratamentos a base de esterco bovino apresentaram os maiores resultados para as variáveis estudadas, seguido dos

\section{REFERÊNCIAS BIBLIOGRÁFICAS}

ALFAIA, S.S.; AYRES, M.I.C. Efeito de doses de nitrogênio, fósforo e potássio em duas cultivares de cupuaçu, com e sem semente, na região da Amazônia Central. Revista Brasileira de Fruticultura, Jaboticabal, v.26, n.2, p.320-325, 2004.

ARAUJO. Q. R.; PAIVA.A. Q. In: VALLE.R. R. M. Ciência, Tecnologia e manejo do cacaueiro. Itabuna: Vital. 2007. v.1. 467p.

BARBOSA. A.L. Nutrição e adubação de plantas cultivadas em substrato. Viçosa, M.G: UFV. p.226-235. 2004.

\section{BARROS, L.E.O.; LIBERALINO FILHO, J.} Composto orgânico sólido e em suspensão na cultura do feijão-mungo-verde (Vigna radiatal, wilkzeck). Revista Verde, Mossoró, v.3, n.1, p.114-122, 2008. tratamentos com caroços de açaí triturados e palha de arroz;

Os caroços de açaí triturado e palha de arroz apresentaram-se como uma alternativa para a produção de biofertilizantes combinados com esterco bovino fresco.

BRASIL. Ministério da Agricultura e Reforma Agrária. Regras para análise de sementes. Brasília: SNDA/ DNDV/CLAV, 1992.

BRITO. M. E. B. et al. Rendimento e qualidade da fruta do maracujazeiro amarelo adubado com potássio, esterco de frango e de ovino. Revista Brasileira de Fruticultura, Jaboticabal, v. 27, n. 3, p. 260-263, 2005.

CAMARGO. S. C. J. O. et al. Desenvolvimento vegetativo de frutíferas em função das relações nitrogênio, potássio, cálcio e magnésio aplicados ao solo. Anais do Curso de Pós-Graduação em Manejo de Solo e Água. Areia. v.23. p.1-11. 2005.

CARRIJO. O. A. et al. Fibra da casca de arroz como substrato agrícola. Revista 
Horticultura Brasileira. Brasília. v.20. n.4. p.533-535. 2002.

CAVALCANTE, L.F. et al. Água salina e esterco bovino líquido na formação de mudas de goiabeira cultivar paluma. Revista

Brasileira de Fruticultura, Jaboticabal, v. 32, n. 01, p.251-261, 2010.

CRUZ, D. E. Secagem e germinação de sementes de cupuaçu (Theobroma grandiflorum (Wiild. Ex Spreng.) Schum. STERCULIACEAE). Revista Brasileira de Sementes, v. 29, n. 3, p. 197-201, 2007.

DAROLT. M. R. Agricultura orgânica: inventando o futuro. Londrina: IAPAR. 2004. 250p.

DELEITO, C. S. R. et al. Sucessão microbiana durante o processo de fabricação do biofertilizante Agrobio. In: FERTBIO REUNIÃO BRASILEIRA DE FERTILIZANTE DO SOLO E NUTRIÇÃO DE PLANTAS; XXIV., 2000, Santa Maria, RS, Anais... Santa Maria: UFSM, 2000.

EMBRAPA AMAZÕNIA ORIENTAL. Programa de melhoramento genético e de adaptação de espécies vegetais para a Amazônia Oriental. Belém, 1999. 137p.

EMBRAPA. Centro Nacional de Pesquisa de Solos. Manual e métodos de análises de solo. 2 ed. Rio de Janeiro, 212p. 2011.

FERREIRA, M. G. R. et al. Morfologia foliar de Theobroma grandiflorum Schum. Ciência Rural, v. 38, n. 2, p. 530-533, 2007.

GALBIATTI, J.A. et al. Efeitos de diferentes doses e épocas de aplicação de efluente de biodigestor e da adubação mineral em feijoeiro-comum (Phaseolus vulgaris L.) submetido a duas lâminas de água por irrigação por sulco. Científica, Jaboticabal, v.24, n.1, p.63- 74, 1996.

KIEHL. E.J. Manual de Compostagem: $3^{\circ}$ edição. Piracicaba 2002.171 pg.

LIMA. M. I. P. M.; SOUZA. A. G. C. Diagnose das principais doenças do cupuaçuzeiro (Theobroma grandiflorum (Willd. Ex Spreng.) Schum.) e seu controle. Manaus: EMBRAPA-CPAA. 2006. 18p.

LORENZI, H. Árvores Brasileiras: Manual de identificação e cultivo de plantas Arbóreas nativas do Brasil. São Paulo: Nova Odessa, 2000.

MACKERETH. F J.H. et al. Water Analysis: Some revised methods for Limnologists. Freshwater Biological Association Cumbria. 121 p. 1978.

MAGRINI. P. G. et al. Comportamento de frutíferas do Estado de Alagoas. Revista Verde de Agroecologia e Desenvolvimento Sustentável (Mossoró - RN - Brasil) v.5. n.2. p. 54 - 63. 2009.

MALAVOLTA. E. ABC da adubação. São Paulo. Ed. Agronômica Ceres. $5^{\text {a }}$ ed. 292 p. 1989.

MALAVOLTA. E. et al. Avaliação do estado nutricional das plantas: princípios e aplicações. 2.ed. Piracicaba: POTAFOS. 319; 1997.

MEDEIROS, M.B. et al. Uso de biofertilizantes líquidos no manejo ecológico de pragas agrícolas. In: ENCONTRO TEMÁTICO MEIO AMBIENTE E EDUCAÇÃO AMBIENTAL DA UFPB, II., 2003, João Pessoa, PB. Anais... João Pessoa, UFPB, 2003. p.19-23.

NOMURA, E. S. et al. Crescimento de mudas micropropagadas da bananeira cv.

Nanicão em diferentes substratos e fontes de fertilizante. Acta Scientiarum Agronomy, v. 30, n. 3, p. 359-363, 2008.

SANTOS. A. C. V. dos. Efeitos nutricionais e fitossanitários do biofertilizante liquido a nível de campo. Revista Brasileira de Fruticultura. v.13. n 4. p. 275 - 279. 1991.

SANTOS. A.C.U. Biofertilizante líquido: o defensivo agrícola da natureza. Niterói: EMATER-RJ. 1992. 16p. 
SOUZA. H.U.; RESENDE E SILVA. C. R.; CARVALHO. J.G.; MENEGUCCI. J.L.P. Nutrição de frutíferas em função de substratos e doses de superfosfato simples. Revista Ciência e Agrotecnologia. Lavras. v.24. p.64-73. 2005.

SOUZA. J. L.; RESENDE. P. Manual de fruticultura orgânica. Viçosa: Aprenda fácil. 564p. 2003.
STATISTICA. Statistica 7.0. EUA Software. Tucksa: StatSof. 2005.

VILELA. G. et al. Avaliação da parte aérea de mudas frutíferas produzidas sob uma perspectiva agroecológica.In: Congresso Brasileiro de fruticultura. Porto Alegre. Anais... Porto Alegre: EMATER: RSASCAR. 2003. 